\title{
Intraductal papilloma of the sublingual gland imitating a ranula
}

\author{
Dimitris Tatsis ${ }^{1}$, Nikolaos Kechagias ${ }^{2}$, Antonios Tsekos ${ }^{2}$, Georgios Petrakis ${ }^{3}$, Konstantinos Vahtsevanos $^{4}$ \\ ${ }^{I}$ Department of Oral and Maxillofacial Surgery, General Hospital of Thessaloniki "G. Papanikolaou”, \\ ${ }^{2}$ Department of Oral and Maxillofacial Surgery, St. Lukes Hospital, Pylaia, ${ }^{3}$ Pathologist, Private Practice, \\ ${ }^{4}$ Department of Oral and Maxillofacial Surgery, Aristotle University of Thessaloniki, Thessaloniki, Greece
}

\begin{abstract}
J Korean Assoc Oral Maxillofac Surg 2022;48:59-62)
Ductal papillomas are rare benign lesions of the salivary glands with two distinctive types, intraductal and inverted. The rarest anatomical location where intraductal papillomas occur is the major salivary glands. In the present study, we report an intraductal papilloma of the left sublingual gland with the relevant clinical, imaging, and histopathological findings and postoperative follow-up. To our knowledge, this is the fourth case of an intraductal papilloma of a sublingual gland to be reported in the international literature. We present this rare case because of its clinical and radiological imitation of a ranula.
\end{abstract}

Key words: Intraductal papilloma, Sublingual gland

\section{Introduction}

Ductal papillomas of the salivary glands are rare benign lesions that are sporadically reported. According to the latest World Health Organization (WHO) classification of head and neck tumours ${ }^{1}$, ductal papillomas can be described as intraductal or inverted papillomas. The papillomas described in the literature usually concern minor salivary glands of the lower lip, followed by the buccal mucosa, palate, and tongue. The mean age of appearance is 54 years old (range, 22-77 years), with no sex preference ${ }^{1,2}$. However, there have been cases of papillomas described in children ${ }^{3}$.

Intraductal papillomas have been described in all major salivary glands, with the parotid gland being the most commonly affected gland ${ }^{4}$. Only 3 cases have been described in the sublingual gland ${ }^{5-7}$. Some authors recognize a third type called sialadenoma papilliferum ${ }^{8}$.

\section{Dimitris Tatsis}

Department of Oral and Maxillofacial Surgery, General Hospital of Thessaloniki “G. Papanikolaou”, G. Papanikolaou Ave, Exochi 57010, Greece

TEL: +30-6932611752

E-mail:dtatsis@outlook.com

ORCID: https://orcid.org/0000-0003-2508-0396

(c) This is an open-access article distributed under the terms of the Creative Commons Attribution Non-Commercial License (http://creativecommons.org/ licenses/by-nc/4.0/), which permits unrestricted non-commercial use, distribution, and reproduction in any medium, provided the original work is properly cited.

Copyright (C) 2022 The Korean Association of Oral and Maxillofacial Surgeons.
The aim of this report is to present a rare case of intraductal papilloma of the sublingual gland, imitating both clinically and radiologically a ranula.

\section{Case Report}

A 53-year-old male patient without any prior medical history presented with a painless, gradually increasing in size cystic lesion of the left sublingual gland, and a clinical diagnosis of a possible ranula was established.(Fig. 1) The cystic lesion was approximately $3 \mathrm{~cm} \times 2.5 \mathrm{~cm}$. Magnetic resonance imaging (MRI) was performed. The MRI revealed a plunging cystic lesion on the floor of the mouth associated with the sublingual gland. The T2-weighted images had a high signal of the cystic lesion.(Fig. 2) According to the imaging and clinical features of the lesion, a diagnosis of a plunging ranula was rendered, and the patient was scheduled for operation. Two treatment options were considered, marsupialisation and total removal of the cystic lesion. The latter was chosen as the preferred treatment of choice, and the cystic lesion along with part of the left sublingual gland was excised under general anaesthesia. No rupture of the capsule or damage of the lingual or sublingual nerves occurred.(Fig. 3)

Follow-up was uneventful, and a postoperative MRI was performed to exclude any remaining lesion.(Fig. 4)

Macroscopically, we received a cystic lesion with dimen- 


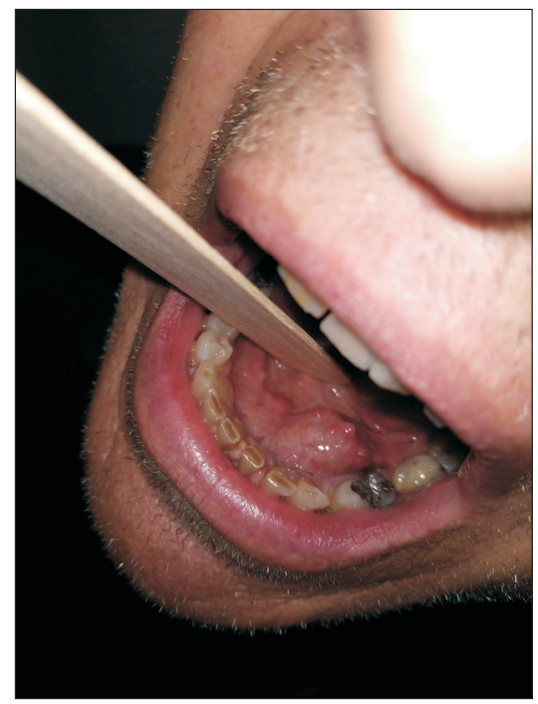

Fig. 1. Preoperative photograph of the lesion.

Dimitris Tatsis et al: Intraductal papilloma of the sublingual gland imitating a ranula. $J$ Korean Assoc Oral Maxillofac Surg 2022

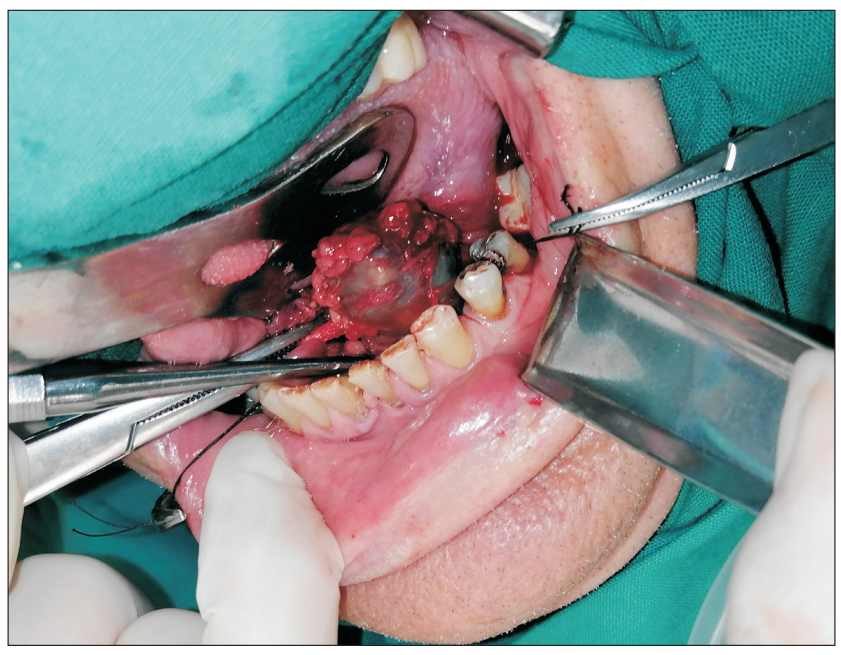

Fig. 3. Intraoperative photograph during the resection of the lesion. Dimitris Tatsis et al: Intraductal papilloma of the sublingual gland imitating a ranula. $J$ Korean Assoc Oral Maxillofac Surg 2022
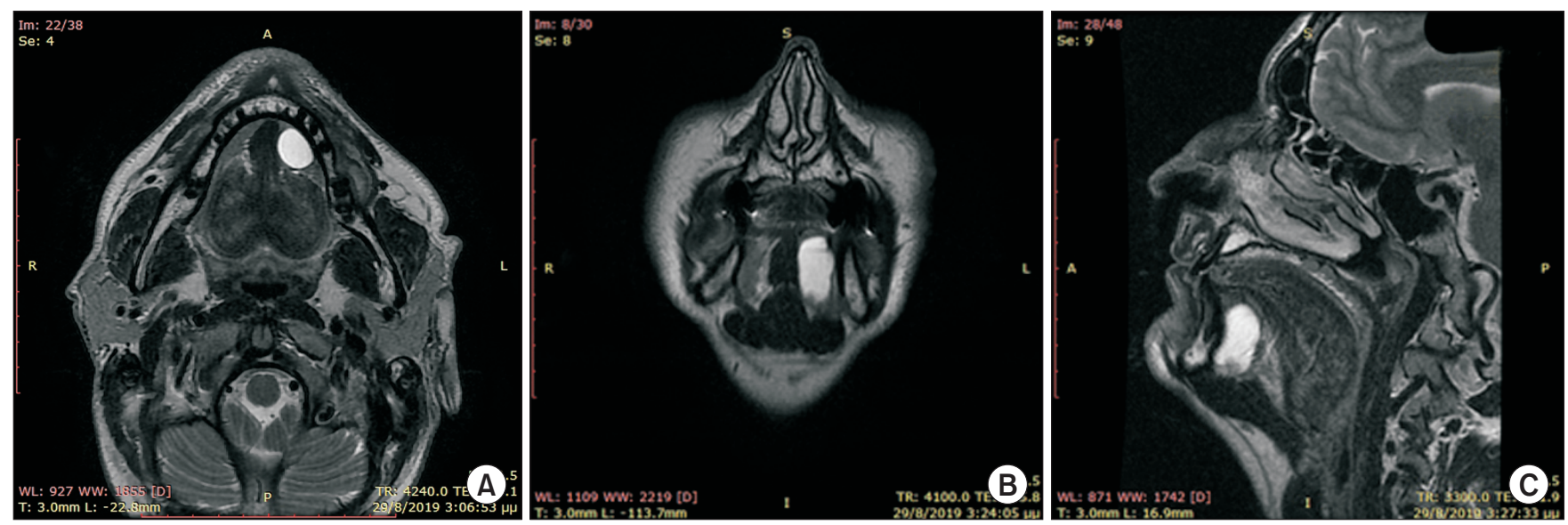

Fig. 2. Preoperative magnetic resonance imaging, T2-weighted image. A. Axial plane. B. Coronal plane. C. Sagittal plane. Dimitris Tatsis et al: Intraductal papilloma of the sublingual gland imitating a ranula. J Korean Assoc Oral Maxillofac Surg 2022
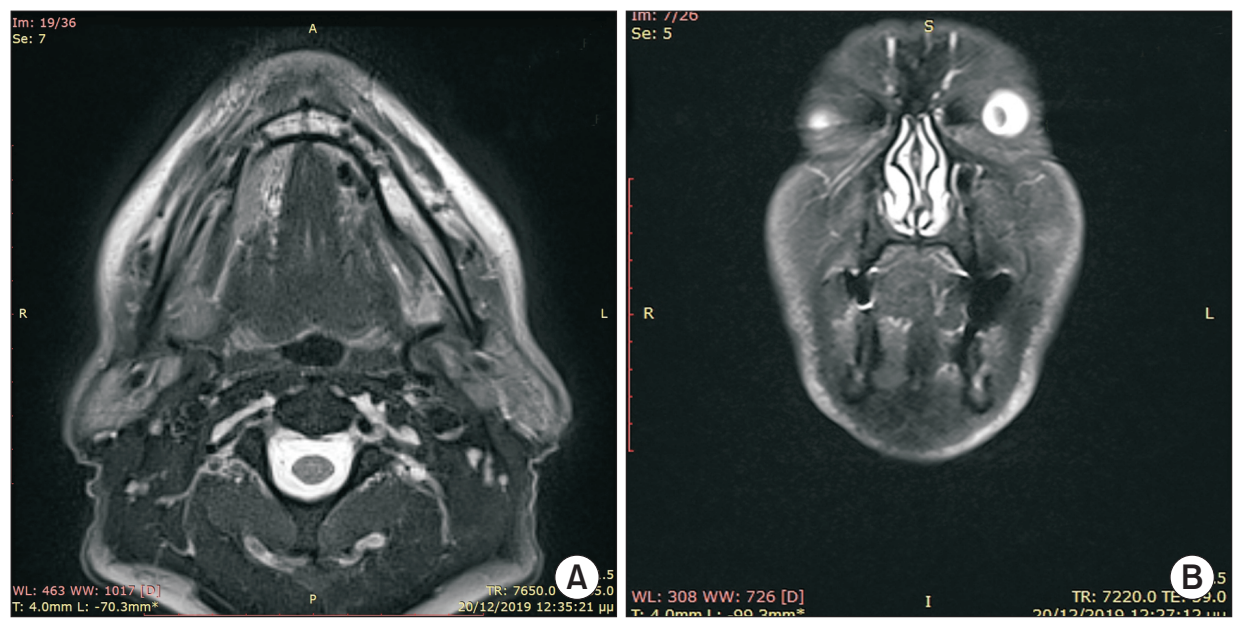

Fig. 4. Postoperative magnetic resonance imaging. A. Axial plane. B. Coronal plane.

Dimitris Tatsis et al: Intraductal papilloma of the sublingual gland imitating a ranula. J Korean Assoc Oral Maxillofac Surg 2022 

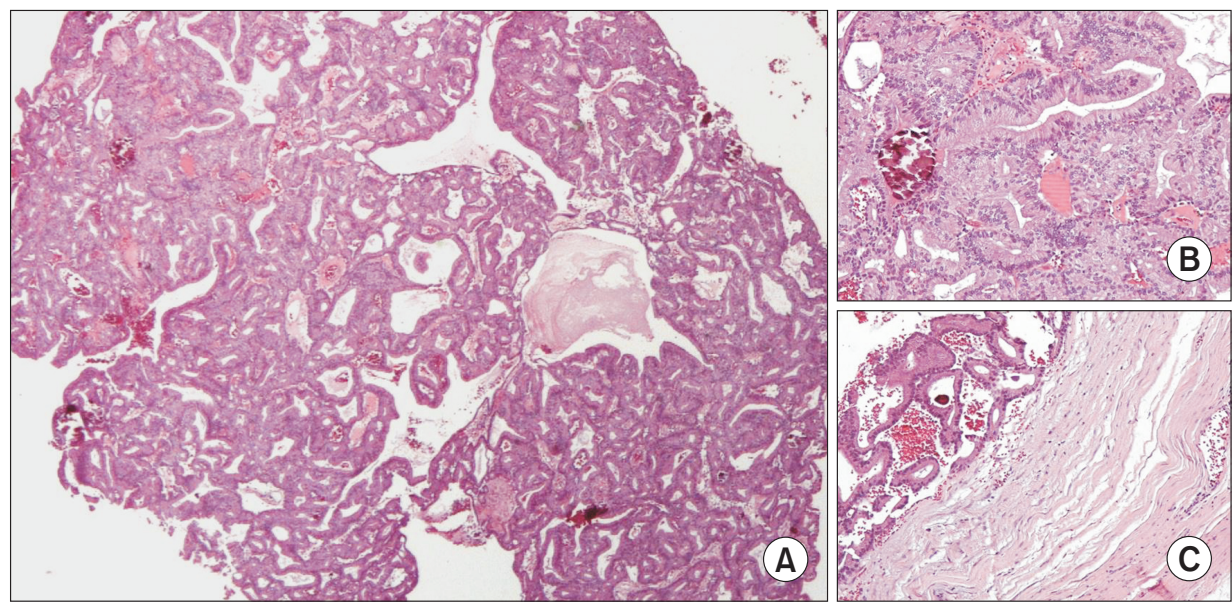

Fig. 5. Histopathological findings. A. Panoramic view of the intraductal papilloma arranged in tubular and papillary formations with thin fibrovascular cores (H\&E staining, $\times 40)$. B. The neoplastic cells are of medium size, cylindrical, with eosinophilic cytoplasm and ovoid nuclei, and without atypia or mitotic activity (H\&E staining, $\times 200$ ). C. There was no infiltration of the neoplastic cells in the ductal wall (H\&E staining, $\times 200)$.

Dimitris Tatsis et al: Intraductal papilloma of the sublingual gland imitating a ranula. J Korean Assoc Oral Maxillofac Surg 2022 sions $3.3 \mathrm{~cm} \times 2.6 \mathrm{~cm} \times 1.5 \mathrm{~cm}$. At dissection, the cystic lesion was unilocular with a wall thickness of $0.1 \mathrm{~cm}$. We identified a small white nodule on the wall (with greatest diameter of 0.5 $\mathrm{cm})$. We also received an unremarked specimen of the sublingual gland, with dimensions $2.7 \mathrm{~cm} \times 1.5 \mathrm{~cm} \times 0.5 \mathrm{~cm}$. Microscopically, we found that the nodule was a neoplasm inside a cystically dilated salivary duct. The neoplastic cells were of medium size, cylindrical, with eosinophilic cytoplasm and ovoid nuclei, and without atypia or mitotic activity. The neoplastic cells were arranged in tubular and papillary formations with thin fibrovascular cores. Few psammoma bodies could be observed. The wall of the cystically dilated duct consisted of one layer of cylindrical cells without atypia or mitotic activity.(Fig. 5. A, 5. B) We also found areas of erosion of the surface epithelium of the cystic wall, as well as areas within the wall with moderate chronic inflammation, fibrosis, calcifications, and hemosiderin deposition. We found no infiltration of neoplastic cells in the ductal wall.(Fig. 5 . C) The second specimen with the rest of the sublingual gland showed no pathological findings. We reached the diagnosis of intraductal papilloma of the sublingual gland. The usual differential diagnosis of such a lesion also includes inverted intraductal papilloma, papillary cystadenoma, and low-grade papillary adenocarcinoma ${ }^{8}$. Taking under consideration all of the above histological features, we reached our final diagnosis ${ }^{1}$.

\section{Discussion}

Ductal papillomas of the salivary glands, according to the WHO classification of head and neck tumours ${ }^{1}$, are painless submucosal masses that have a slow growth. Their aetiology is not well known, although a correlation with HPV virus has been reported ${ }^{9}$. Another possible association is with masticatory trauma ${ }^{10}$.
Definitive pathological diagnosis of an intraductal papilloma is based on their monocystic morphology, the proliferation of the ductal epithelium with a single layer of columnar cells, and the thin fibrovascular junctions ${ }^{4,11}$.

A recent study by Agaimy et al. ${ }^{12}$ suggests that intraductal papillomas behave at the cellular level similarly to pancreatic intraductal papillary mucinous neoplasms due to an AKT1 kinase mutation. Thus, they suggest the term "intraductal papillary neoplasm" for intraductal papillomas, since their biological behavior can be more similar to their pancreatic counterparts. The same results were also described in a recent study by Nakaguro et al. ${ }^{13}$, where it is stated that an AKT1 E17K mutation was present in most of the nine examined specimens of intraductal papillary mucinous neoplasms of minor salivary glands, and almost half of the examined cases appeared to have HRAS Q61R mutations. BRAF, KRAS, PIK3CA, or GNAS mutations were also examined, but with negative results. The same study also examined in a separate category three intraductal papillomas of minor salivary glands, but they did not find any of the aforementioned mutations ${ }^{13}$.

Intraductal papillomas are slowly growing, benign lesions that will eventually obstruct the excretion mechanism of saliva. This explains why our patient presented with signs of ranula of the sublingual gland. Similar clinical signs and symptoms have been described by other reports. Aikawa et al. ${ }^{5}$ reported a painless formation with bluish color and normal mucosa but with normal saliva secretion, while Hara et al. reported a growing mass in the floor of the mouth and Nagao et al. ${ }^{6}$ a painless mass. This may be the case in the major salivary glands, but in the minor glands, which are the more common site, the clinical presentation is less prominent, with a small painless nodule and without any other symptoms. In the study published by Hara et al. ${ }^{7}$, fine needle aspiration 
was performed in a lesion of a sublingual gland that was suspected clinically and radiographically to be adenoid cystic carcinoma. The cytological findings directed the diagnosis to basal cell adenoma, but the pathological examination after excision set the final diagnosis as an intraductal papilloma.

Excision is the treatment of choice, and the literature shows that this is a definitive treatment, since no relapse has been recorded $^{4}$. All 3 of the other cases of intraductal papillomas in the sublingual glands were surgically excised with no relapse. A total sublingual glandectomy was performed by Hara et al. ${ }^{7}$, whereas Nagao et al. ${ }^{6}$ performed a partial glandectomy and Aikawa et al. ${ }^{5}$ an excisional biopsy. There is a malignant variant described by Nagao et al. ${ }^{6}$, which was found in the parotid gland of a 70-year-old male. No recurrence was described after a subtotal parotidectomy and postoperative radiotherapy in a six-year follow-up period. Interestingly, this comes in contrast to their counterpart in the breast, since the latter has a high incidence of recurrence ${ }^{10}$.

To our knowledge, there have been 3 intraductal papillomas of the sublingual gland reported to date. Our case is the fourth, proving the rarity of this condition. Since this is a case where the clinical and radiological findings pointed to a possible diagnosis of a ranula, proper pathologic examination is of paramount importance. Other papillary lesions with a higher prevalence and a higher incidence of malignant transformations need to be in the differential diagnosis and must be ruled out before setting this diagnosis.

Recurrence of a plunging ranula of the floor of the mouth after marsupialization should be considered as an indication of a possible misdiagnosed intraductal papilloma of the sublingual gland.

\section{ORCID}

Dimitris Tatsis, https://orcid.org/0000-0003-2508-0396

Nikolaos Kechagias, https://orcid.org/0000-0002-8908-6140

Antonios Tsekos, https://orcid.org/0000-0001-9354-1620

Georgios Petrakis, https://orcid.org/0000-0003-4529-6784

Konstantinos Vahtsevanos, https://orcid.org/0000-0002-3758-8616

\section{Authors' Contributions}

D.T. wrote the manuscript. A.T. and N.K. collected the data and conducted the literature review. G.P. contributed to the histopathological specimens and provided the pathology report. K.V. critically revised the manuscript and had the overall care of the publication.

\section{Conflict of Interest}

No potential conflict of interest relevant to this article was reported.

\section{References}

1. El-Naggar AK, Chan JKC, Grandis JR, Takata T, Slootweg PJ, eds. WHO classification of head and neck tumours. 4th ed. Lyon: IARC (International Agency for Research on Cancer); 2017.

2. Swerdlow SH, Campo E, Harris NL, Jaffe ES, Pileri SA, Stein H, et al. WHO classification of tumours of haematopoietic and lymphoid tissues. 4th ed. Lyon: IARC; 2008:202-8.

3. Choi G, Choi CS, Choi JS, Choi JO. Intraductal papilloma of the parotid gland in a child. Otolaryngol Head Neck Surg 2001;125: 280. https://doi.org/10.1067/mhn.2001.115666

4. Brannon RB, Sciubba JJ, Giulani M. Ductal papillomas of salivary gland origin: a report of 19 cases and a review of the literature. Oral Surg Oral Med Oral Pathol Oral Radiol Endod 2001;92:6877. https://doi.org/10.1067/moe.2001.115978

5. Aikawa T, Kishino M, Masuda T, Isomura ET, Tanaka S, Namikawa $\mathrm{M}$, et al. Intraductal papilloma arising from sublingual minor salivary gland: case report and immunohistochemical study. Oral Surg Oral Med Oral Pathol Oral Radiol Endod 2009;107:e34-7. Erratum in: Oral Surg Oral Med Oral Pathol Oral Radiol Endod 2009;108:475. https://doi.org/10.1016/j.tripleo.2009.01.019

6. Nagao T, Sugano I, Matsuzaki O, Hara H, Kondo Y, Nagao K. Intraductal papillary tumors of the major salivary glands: case reports of benign and malignant variants. Arch Pathol Lab Med 2000;124: 291-5. https://doi.org/10.5858/2000-124-0291-IPTOTM

7. Hara H, Oyama T, Omori K, Misawa T, Kasai H, Kimura M, et al. Fine needle aspiration cytology of an intraductal papilloma originating in a sublingual gland. A case report. Acta Cytol 1999;43: 457-63. https://doi.org/10.1159/000331100

8. Wenig BM. Atlas of head and neck pathology. 3rd ed. Philadelphia (PA): Elsevier; 2015:908-14, 490-3.

9. Infante-Cossio P, Gonzalo DH, Hernandez-Gutierrez J, BorreroMartin JJ. Oral inverted ductal papilloma associated with condyloma acuminata and HPV in an HIV+ patient. Int J Oral Maxillofac Surg 2008;37:1159-61. https://doi.org/10.1016/j.ijom.2008.07.016

10. Hellquist H, Paiva-Correia A, Vander Poorten V, Quer M, Hernandez-Prera JC, Andreasen S, et al. Analysis of the clinical relevance of histological classification of benign epithelial salivary gland tumours. Adv Ther 2019;36:1950-74. https://doi.org/10.1007/s12325019-01007-3

11. Ramaswamy P, Khaitan T, Anuradha A, Kumar BP, Sudhakar S. Intraductal papilloma: atypical presentation. Case Rep Dent 2013; 2013:652728. https://doi.org/10.1155/2013/652728

12. Agaimy A, Mueller SK, Bumm K, Iro H, Moskalev EA, Hartmann A, et al. Intraductal papillary mucinous neoplasms of minor salivary glands with AKT1 p.Glu17Lys mutation. Am J Surg Pathol 2018; 42:1076-82. https://doi.org/10.1097/PAS.0000000000001080

13. Nakaguro M, Urano M, Ogawa I, Hirai H, Yamamoto Y, Yamaguchi $\mathrm{H}$, et al. Histopathological evaluation of minor salivary gland papillary-cystic tumours: focus on genetic alterations in sialadenoma papilliferum and intraductal papillary mucinous neoplasm. Histopathology 2020;76:411-22. https://doi.org/10.1111/his.13990

How to cite this article: Tatsis D, Kechagias N, Tsekos A, Petrakis G, Vahtsevanos K. Intraductal papilloma of the sublingual gland imitating a ranula. J Korean Assoc Oral Maxillofac Surg 2022;48:59-62. https://doi.org/10.5125/jkaoms.2022.48.1.59 